\title{
Analyse élastohydrodynamique des joints à lèvre avec micro-ondulations
}

\author{
Mohamed HajJam ${ }^{\mathrm{a}}$ et Dominique Bonneau \\ Université de Poitiers Laboratoire de Mécanique des Solides (UMR 6610), SP2MI, Bd Marie et Pierre Curie, BP 30179, \\ 86962 Futuroscope Chasseneuil Cedex, France
}

Reçu le 20 février 2003 ; accepté le 16 juin 2003

\begin{abstract}
Résumé - L' étude d'un joint à lèvre, basée sur une analyse élastohydrodynamique, s'appuie sur un modèle original très proche de la réalité. Elle permet de prédire la plupart des caractéristiques de fonctionnement d'un joint telles que l'épaisseur du film lubrifiant, le couple de frottement, les zones de contact et de cavitation, la pression hydrodynamique ainsi que le débit de pompage inverse.
\end{abstract}

Mots clés : Joints à lèvre / élastohydrodynamique / éléments finis / équation de Reynolds / cavitation

\begin{abstract}
Elastohydrodynamic analysis of lip seals with micro-ondulations. In the present study, a realistic model of lip seal is developed. An ElastoHydrodynamic analysis is used to generate predictions of such seal operating characteristics as thickness distribution, hydrodynamic pressure, contact and cavitation area, friction torque and reverse leakage pumping.
\end{abstract}

Key words: Lip Seals / Elastohydrodynamic / finite elements / cavitation / Reynolds equation

\section{Introduction}

Dès les années 1950, il a été établi que sous la lèvre d'un joint d'étanchéité en élastomère se développe un film lubrifiant qui évite à celle-ci un frottement et une température prohibitifs [1] ; la naissance et le maintien de ce film mince sont dus à la rugosité de la lèvre [2]. Bien que depuis de nombreux travaux expérimentaux aient été réalisés avec succès, aucune modélisation numérique n'avait été développée avant les années 1990 pour prédire le comportement mécanique de cet organe. Cette absence de modélisation s'expliquait par le manque de connaissances des phénomènes physiques qu'engendre l'utilisation des joints à lèvre, et surtout par le manque d'outils de calcul appropriés. Ces deux handicaps n'ont été surmontés que durant ces dix dernières années et depuis lors de nombreux travaux de modélisation ont été publiés. Parmi ces travaux on peut citer ceux de Müller [3,4] et Horve [5] qui se sont intéressés au débit de pompage inverse, c'est-à-dire au refoulement du fluide des pressions les plus faibles vers les pressions les plus fortes, ainsi que ceux qui ont montré la forte corrélation entre la micro-géométrie de la lèvre, la charge supportée et

a Auteur correspondant :

hajjam@iutang.univ-poitiers.fr le débit de fuite [6-8]. Pour expliquer le rôle joué par l'état de surface de la lèvre dans la prévention du débit de fuite, certains auteurs ont modélisé les irrégularités de la surface soit par des aspérités soit par des micro ondulations régulières ou quasi aléatoires, disposées perpendiculairement à la circonférence [3,6-10]. Stakenborg [11] a montré que l'effet de la tension superficielle contribue à renforcer l'efficacité de l'étanchéité du joint à lèvre. Des analyses plus complètes élastohydrodynamiques et thermoélastohydrodynamiques ont été proposées Salant et al. [12-14]. Ces modèles, qui intègrent la base de connaissances de ces dix dernières années, confirment le rôle de la rugosité de la lèvre sur la naissance et le maintien du film lubrifiant, l'influence de la tension superficielle du lubrifiant sur l'efficacité de l'étanchéité, et donnent des prédictions numériques proches des mesures expérimentales de plusieurs grandeurs caractéristiques telles que l'épaisseur du film lubrifiant, le couple de frottement, le débit de pompage et de fuite et la température de la lèvre. Cependant, si l'on veut réduire les écarts observés, il faut d'une part intégrer toutes les connaissances actuelles de ce domaine, et d'autre part, améliorer les modèles proposés qui restent assez limités du point de vue de la modélisation de la structure du joint. En effet, ils supposent que la partie compliante du joint à lèvre a un comportement mécanique bidimensionnel 


\section{Nomenclature}

\begin{tabular}{|ll|}
\hline$b$ & longueur de la cellule représentant la lèvre du joint \\
$C i j$ & matrice de compliance à la pression \\
$D$ & variable universelle (pression dans la zone active et remplissage dans la zone inactive) \\
$E_{j}\left(\Omega_{e}\right)$ & évaluation de la fonctionnelle au noud $j$ de l'élément $\Omega_{e}$ \\
$f(x, y)$ & forme analytique du défaut de la surface de la lèvre \\
$F$ & fonction de contrôle $(F=1$ dans la zone active $; F=0$ dans la zone inactive) \\
$h$ & épaisseur du film lubrifiant \\
$h_{0}$ & amplitude du défaut de la surface de la lèvre \\
$h_{3}$ & hauteur de la partie 3D de la lèvre \\
$N$ & nombre de périodes du défaut de la surface de la lèvre \\
$P$ & pression hydrodynamique \\
$r$ & remplissage \\
$R$ & rayon de l'arbre \\
$T_{k i}$ & matrice de compliance au cisaillement \\
$U$ & vitesse linéaire de l'arbre \\
$W, W$ & fonctions de pondération \\
$x$ & coordonnée circonférentielle \\
$y$ & coordonnée axiale \\
$\delta$ & déplacement circonférentielle dû au cisaillement \\
$\lambda$ & longueur d'onde du défaut de la surface de la lèvre \\
$\mu$ & viscosité dynamique du lubrifiant \\
$\tau_{x y i}$ & contrainte de cisaillement locale \\
$\rho$ & masse volumique du mélange lubrifiant-gaz \\
$\rho_{0}$ & masse volumique du lubrifiant \\
$\Omega, \partial \Omega$ & respectivement domaine et frontière du domaine d'étude \\
$\Omega_{e}$ & domaine d'un élément (domaine élémentaire) \\
\hline
\end{tabular}

(axisymétrique), ce qui est loin d'être le cas de l'arête de la lèvre.

Le présent travail propose d'améliorer de manière significative le modèle élastohydrodynamique pour le rendre plus réaliste, avec pour l'arête du joint un comportement élastique tridimensionnel, et pour l'hydrodynamique une gestion fine de la cavitation conforme à la théorie de J.F.O. (Jacobson et Floberg [15] et Olsson [16]).

\section{Position du problème}

La section droite d'un joint à lèvre a la forme proposée sur la figure 1 .

Lorsqu'il est libre et sans contrainte, le diamètre de la lèvre d'étanchéité est toujours plus petit que le diamètre de l'arbre. La différence entre ces deux côtes est le préserrage. Ce préserrage écrase la lèvre d'étanchéité lors du montage et fait naître une force radiale dont l'effet est de comprimer l'arête d'étanchéité contre l'arbre. L'importance de cette force dépend de l'élastomère utilisé, de la température, du profil géométrique de la lèvre d'étanchéité ainsi que de la raideur du ressort.

La force radiale de la lèvre produit, compte tenu de sa forme géométrique (angle de la lèvre) et de l'intervalle d'action du ressort, une répartition asymétrique de la pression de serrage $p_{s}$ (pression avec montée rapide du côté huile et lente diminution du côté air) comme le montre le détail (A) de la figure 1.
L'approche utilisée pour la modélisation de calcul de structure est nouvelle et originale. En effet, toute la partie compliante du joint n'est pas supposée avoir un comportement élastique axisymétrique, elle est décomposée en deux parties ayant des comportements mécaniques différents (Fig. 2).

La partie (1), dont la hauteur h3 est à fixer (dépend du joint à étudier), est supposée avoir un comportement élastique tridimensionnel, tandis que la partie (2) a un comportement axisymétrique. La forme de la partie (1) en contact avec l'arbre tournant peut être considérée comme une bande dont la longueur $b$ dépend du préserrage. Le joint étant supposé centré, l'examen de l'état de surface de cette bande montre une rugosité périodique dans la direction circonférentielle, de longueur d'onde $\lambda$. L'analyse du comportement du joint peut se faire sur une seule période de cette bande, et donc sur un rectangle $(\lambda, b)$ que nous nommerons cellule. La complexité de la modélisation a nécessité l'élaboration d'un mailleur spécifique. Ce dernier permet de construire le maillage à partir de la saisie d'une part de coordonnées de points particuliers du joint et de la hauteur $h$ de l'arête et d'autre part des dimensions de la cellule d'étude $(\lambda, b)$.

La figure 3(a,b,c) montre le maillage obtenu. Ainsi, la figure 3 a présente une vue globale du maillage $3 \mathrm{D}-2 \mathrm{D}$; la figure $3 \mathrm{~b}$ pointe la partie 3D (l'arête de la lèvre) ; et enfin la figure $3 \mathrm{c}$ schématise la jonction des maillages 3D-2D. Le calcul de la matrice de compliance nécessaire à l'étude EHD de la structure est effectué en tenant compte des particularités de ce maillage. 

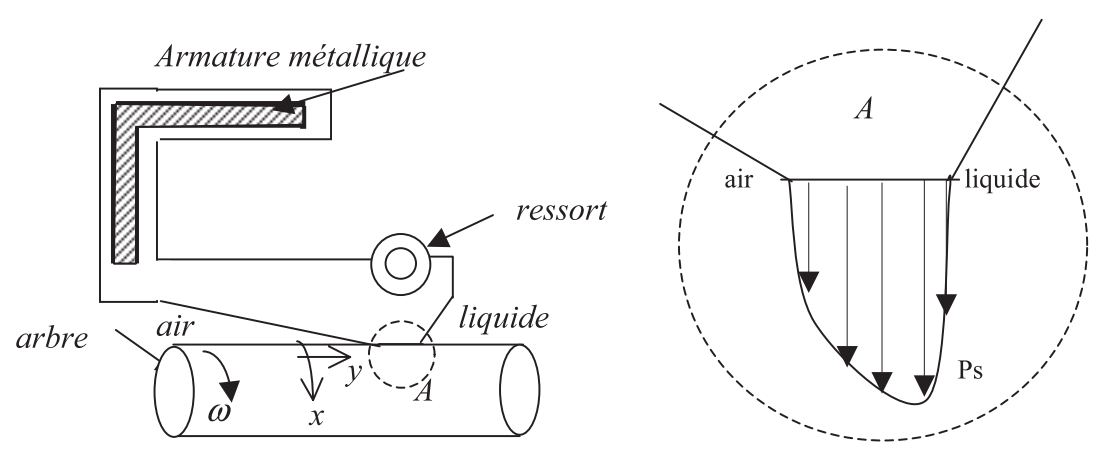

Fig. 1. Schématisation d'un joint à lèvre et détail de $\mathrm{A}$.

Pour le cas présenté par la suite, le préserrageest de $2 \mathrm{~mm}$, ce qui induit pour la cellule une longueur $b=$ $0,1 \mathrm{~mm}$; l'analyse de la périodicité du défaut de la surface de la lèvre donne sa largeur $\lambda=0,0125 \mathrm{~mm}$; le champ de pression statique $\mathrm{p}_{\mathrm{s}}$ qui écrase la lèvre est donné sur la figure 4 et enfin, la hauteur de la partie 3D de la lèvre est fixée à $h_{3}=0,5 \mathrm{~mm}$.

La résolution du problème élastohydrodynamique se fait sur le maillage 2D de cette cellule.

\section{Hypothèses et équations}

Les hypothèses retenues sont :

- partie compliante du joint parfaitement élastique;

- arbre tournant parfaitement lisse;

- joint centré (pas de fouettement).

Après l'élaboration de la matrice de compliance à partir du maillage 3D-2D de la structure (Fig. 3a), on résout l'équation de Reynolds couplée, par le biais de la matrice de compliance, au comportement élastique du joint, tout en gérant la cavitation. La démarche adoptée est la suivante : l'équation de Reynolds sous sa forme stationnaire pour un fluide isovisqueux

$$
\frac{\partial}{\partial x}\left(\rho h^{3} \frac{\partial p}{\partial x}\right)+\frac{\partial}{\partial y}\left(\rho h^{3} \frac{\partial p}{\partial y}\right)=6 \mu U \frac{\partial \rho h}{\partial x}
$$

doit être vérifiée pour les zones actives (zones sous pression). Cette même équation se réduit à (2) pour les zones inactives (zones en cavitation), zones où la pression $p$ est constante :

$$
\frac{\partial(\rho h)}{\partial x}=0
$$

où $\rho$ représente la masse volumique du mélange lubrifiantgaz dû̀ à la rupture du film lubrifiant.

En définissant le remplissage $r$ par :

$$
r=\frac{\rho h}{\rho_{0}}
$$

où $\rho_{0}$ est la masse volumique du lubrifiant, l'équation (2) se transforme en (3):

$$
\frac{\partial r}{\partial x}=0
$$

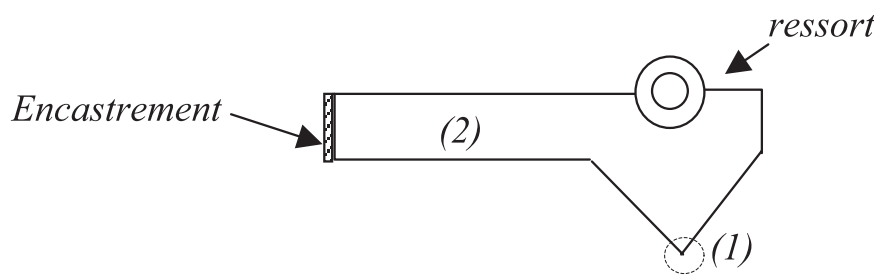

Fig. 2. Schématisation de la partie compliante du joint à lèvre.

Afin de traiter simultanément les équations (1) et (3), elles sont regroupées en une seule (4) en utilisant une variable universelle nommée $\mathrm{D}$ et une fonction $\mathrm{F}$ qui permet de distinguer les différentes zones.

C'est dans les zones actives que la pression s'établit et que l'équilibre avec la charge appliquée se réalise. Dans les zones inactives, il y aura conservation du débit massique. Nous avons donc :

$$
\begin{aligned}
& \frac{\partial}{\partial x}\left(\rho h^{3} \frac{\partial F D}{\partial x}\right)+\frac{\partial}{\partial y}\left(\rho h^{3} \frac{\partial F D}{\partial y}\right)= \\
& 6 \mu U \frac{\partial}{\partial x}[(h+(1-F) D)]
\end{aligned}
$$

- pour les zones actives :

$$
\left\{\begin{array}{l}
D=p \geq 0 \\
F=1
\end{array}\right.
$$

- pour les zones inactives :

$$
\left\{\begin{array}{l}
D=r-h<0 \\
F=0
\end{array}\right.
$$

Pour tout point du film, on devra vérifier la compatibilité entre le signe de $D$ et l'état supposé, actif ou inactif, du film en ce point. Comme nous l'avons déjà montré [17], les conditions aux limites utilisées pour la résolution de cette équation de Reynolds modifiée sont basées sur la séparation des zones actives et inactives, tout en tenant compte des conditions sur les frontières extérieures du domaine considéré.

\section{Formulation éléments finis du problème EHD}

Les équations d'un problème EHD sont l'équation de Reynolds, l'équation d'équilibre de la charge et les 


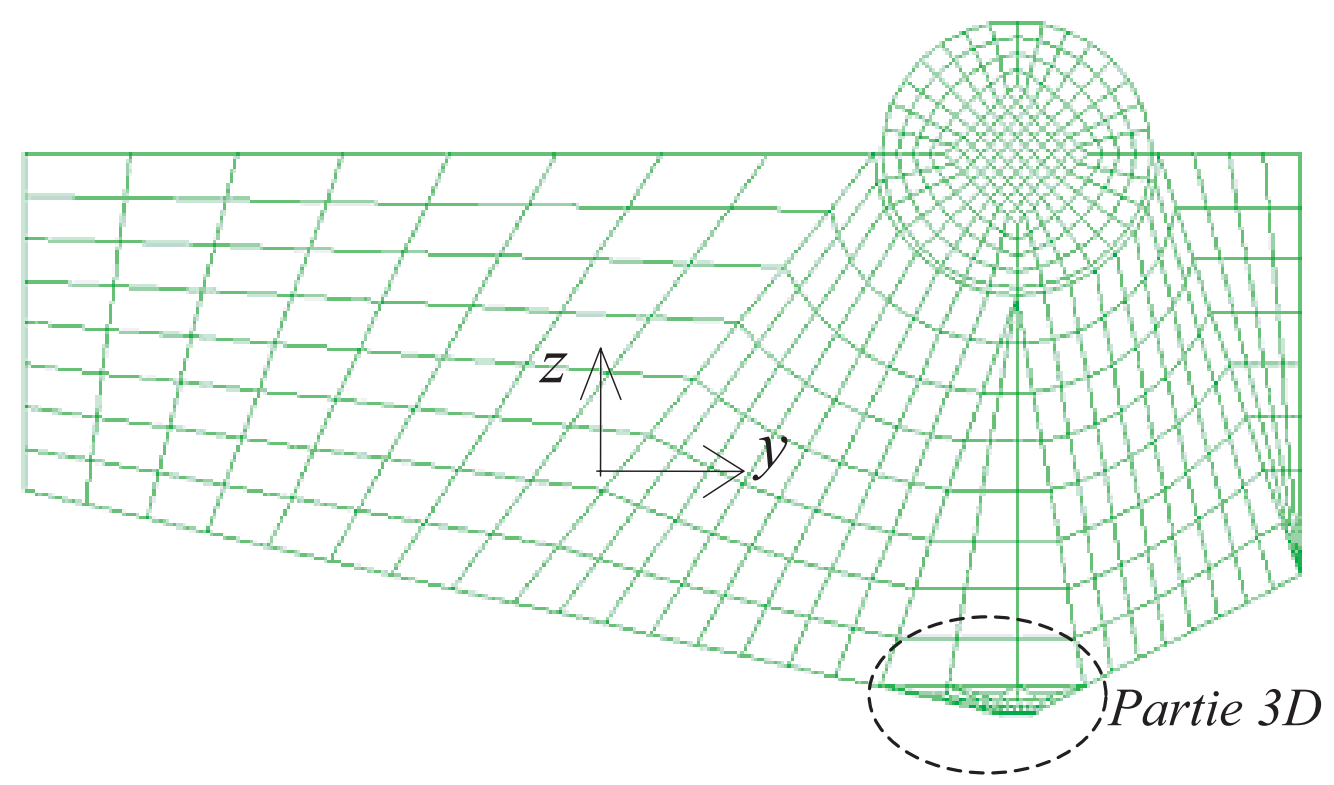

(a)

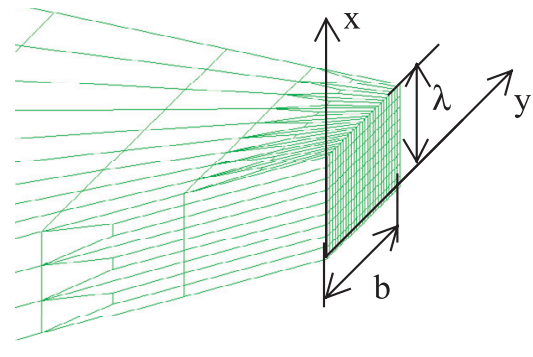

(b)

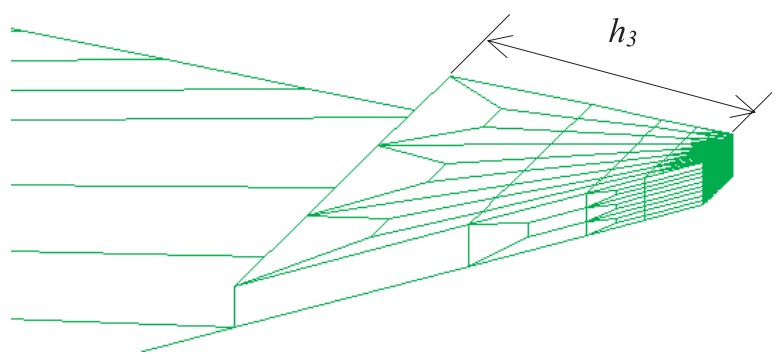

(c)

Fig. 3. (a) Maillage de toute la partie compliante du joint; (b) maillage de la partie 3D, (c) jonction entre le maillage 2D et 3D.

équations de l'élasticité. L'action de la pression sur les surfaces élastiques qui délimitent le film lubrifiant se traduit par la déformation de celles-ci. L'épaisseur h du film doit donc être déterminée simultanément à la pression. La non connaissance a priori de la localisation des zones actives et inactives du film conduit donc à la définition de deux problèmes distincts qui seront résolus alternativement :

1. l'épaisseur du film est connue : on recherche les frontières des zones actives et inactives;

2 . les zones actives et inactives sont connues : on recherche le champ de pression et le champ d'épaisseur qui vérifient l'équation de Reynolds et les équations de l'élasticité.

La recherche des zones actives et inactives est donnée par la résolution de l'équation de Reynolds modifiée (4). La pression et l'épaisseur sont déterminées en considérant l'équation de Reynolds (1) et les relations reliant l'épaisseur du film à la pression. En raison de la similitude entre ces équations, la discrétisation par la méthode des éléments finis est présentée pour l'équation de Reynolds modifiée.
Considérons la fonctionnelle

$$
\begin{array}{r}
E^{*}(D)=\int_{\Omega} W^{*} F\left(-\frac{\partial}{\partial x}\left(\frac{h^{3}}{6 \mu} \frac{\partial D}{\partial x}\right)-\frac{\partial}{\partial y}\left(\frac{h^{3}}{6 \mu} \frac{\partial D}{\partial y}\right)\right. \\
\left.+U \frac{\partial h}{\partial x}-(1-F) U \frac{\partial D}{\partial x}\right) \mathrm{d} \Omega
\end{array}
$$

où $W^{*}$ est une fonction suffisamment différentiable définie $\operatorname{sur} \Omega$.

Nous avons montré par ailleurs [17] qu'une intégration par parties de certains termes, puis l'addition de fonctionnelles complémentaires définies sur les frontières de transition entre zones actives et inactives permettent, d'une part de réduire l'ordre de dérivabilité des fonctions (formulation faible) et d'autre part de rendre naturelles les conditions aux limites de rupture et de reformation du film nécessaires à la localisation de ces zones. Si les conditions aux limites extérieures du domaine portent sur le débit, les termes complémentaires résultant de l'intégration par parties doivent être conservés. Mais généralement pour les joints à lèvre, c'est la pression qui est imposée sur ces frontières. Dans ce cas, afin de ne pas 
faire apparaître ces termes, les fonctions $W$ sont choisies nulles sur la frontière $\partial \Omega$, d'où l'équation (6) :

$$
\begin{array}{r}
E(D)=\int_{\Omega}\left(\frac{h^{3}}{6 \mu}\left(\frac{\partial W}{\partial x} \frac{\partial F D}{\partial x}+\frac{\partial W}{\partial y} \frac{\partial F D}{\partial y}\right)+\right. \\
\left.W U \frac{\partial h}{\partial x}+(1-F) U D \frac{\partial W}{\partial x}\right) \mathrm{d} \Omega
\end{array}
$$

La localisation des différentes zones est obtenue en recherchant les fonctions $D$ qui satisfont aux conditions aux limites sur les frontières extérieures $\partial \Omega$ du film et telles que $E(D)=0$. La non linéarité des équations nécessite un algorithme itératif décrit par la suite.

La discrétisation des équations est basée sur des éléments quadratiques à 8 nœuds. Le domaine $\Omega$ est donc divisé en $n_{e}$ éléments finis isoparamétriques à huit nœuds. Le caractère particulier de l'équation de Reynolds modifiée, lorsqu'elle s'applique aux zones inactives (équation hyperbolique), rend préférable pour sa discrétisation l'usage d'éléments linéaires à quatre nœuds. En effet, afin d'éviter des instabilités numériques, les fonctions poids du processus de Bubnov-Galerkin doivent être décentrées vers l'amont, opération plus aisée avec des éléments linéaires. Chaque élément à 8 nœuds est donc subdivisé en quatre éléments quadrangulaires à quatre nœuds. Selon qu'il s'agit du problème 1 ou 2 , la discrétisation fera appel à l'un ou l'autre maillage, la valeur des paramètres au nœud central étant éventuellement obtenue par interpolation.

Les fonctions d'interpolation $N$ choisies, linéaires ou quadratiques selon le cas, permettent d'interpoler à la fois les variables géométriques et les différents paramètres. Dans le cas du problème 1, la fonctionnelle (6) évaluée au nœud $j$ d'un élément $\Omega_{e}$ s'écrit :

$$
\begin{aligned}
E_{j}\left(\Omega_{e}\right)= & \sum_{m=1}^{n_{p g}}\left(\frac { h _ { m } ^ { 3 } } { 6 \mu } \sum _ { k = 1 } ^ { n n _ { e } } \left(\frac{\partial W_{m j}}{\partial x} \frac{\partial N_{m k}}{\partial x}\right.\right. \\
& \left.+\frac{\partial W_{m j}}{\partial y} \frac{\partial N_{m k}}{\partial y}\right) F_{k} D_{k}+W_{m j} U \frac{\partial h_{m}}{\partial x} \\
& \left.+\sum_{k=1}^{n n e} \frac{\partial W_{m j}}{\partial x} N_{m k}\left(1-F_{k}\right) D_{k}\right) \Delta \Omega_{m}
\end{aligned}
$$

où $m$ est l'un des $n_{p g}$ (en général 4) points de Gauss sur cet élément et $n n_{e}$ le nombre de nœuds par élément (8 ou 4). $W_{m j}$ est la fonction de pondération relative au noud $j$ et $N_{m k}$ est la fonction d'interpolation relative au nœud $k$ appartenant à $\Omega_{e}$; elles sont évaluées au point de Gauss $m . F_{k}$ représente l'état du nœud $k$ et prend la valeur 1 s'il est dans une zone active et 0 dans le cas contraire.

La sommation de l'ensemble des équations (7) écrites sur chacun des $n_{\mathrm{e}}$ éléments du domaine $\Omega$ conduit à un système d'équations algébriques linéaires en $D$. La résolution du système linéaire obtenu donne la solution recherchée.

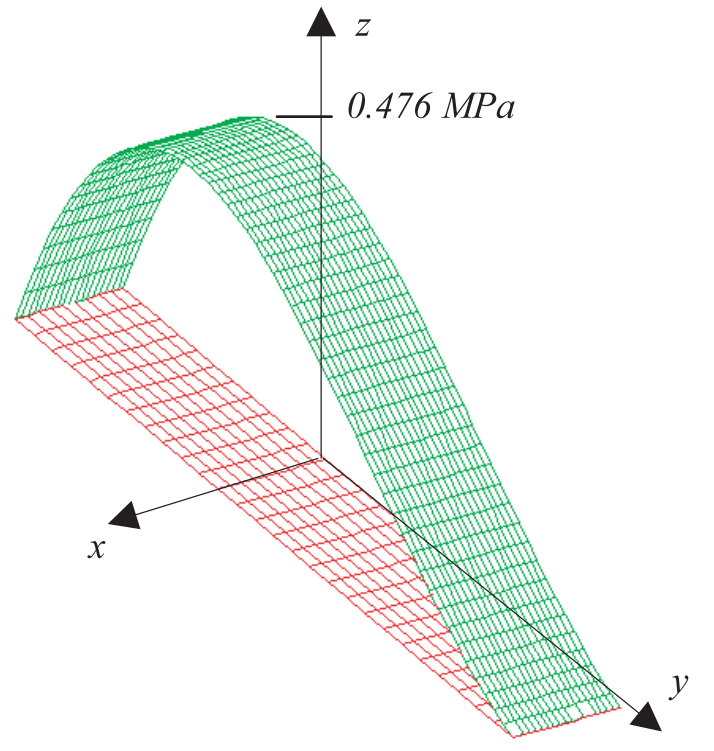

Fig. 4. Répartition de la pression $p_{s}$ due au préserrage.

Lorsque le problème à résoudre est du type 2 , le domaine d'intégration est restreint aux zones actives pour lesquelles on a $F_{k}=1$. En raison de l'élasticité des surfaces, l'épaisseur $h$ dépend de $p$, mais aussi de l'état de surface de la lèvre.

L'épaisseur $h$ du film est donnée par :

$$
h(x, y)=f(x, y)+h_{m}+\eta
$$

avec :

$$
h_{m}=\sum_{k=1}^{n n e} N_{m k} h_{k}=\sum_{k=1}^{n n e} N_{m k} \sum_{i=1}^{n} C_{k i} p_{i}
$$

$h_{m}$ : contribution élastique à l'épaisseur du film; $C_{k i}:$ matrice de compliance; $P_{i}$ : pression nodale; $\eta$ : épaisseur minimale; $f(x, y)$ : fonction mathématique représentant les irrégularités de la surface de la lèvre du joint.

Pour cette dernière, plusieurs modèles ont été testés :

$$
\left\{\begin{array}{l}
f(x, y)=\frac{h_{0}}{2} \cos (2 \pi(x-\delta))(1-\cos (2 \pi N y)) \\
f(x, y)=\frac{h_{0}}{2} \sin (2 \pi(x-\delta)) \sin (2 \pi N y) \\
f(x, y)=\frac{h_{0}}{2} \sin (2 \pi(x-\delta)) \\
f(x, y)=\frac{h_{0}}{2}\left(\cos (2 \pi x)+k \frac{y-0.5}{0.5}\right)
\end{array}\right.
$$

$\delta$ représente le déplacement tangentiel des irrégularités de la surface de la lèvre dû à la contrainte tangentielle et $N$ le nombre de creux et/ou bosses de la rugosité :

$$
\delta=\sum_{k=1}^{n n e} N_{m k} \sum_{i=1}^{n} T_{k i} \tau_{x y i}
$$

Dans ce cas, les équations à résoudre sont non linéaires en $p$. Leur résolution fait appel à un processus de NewtonRaphson. 


\section{Algorithme numérique}

L'algorithme adopté est celui proposé par [17]. Il est adapté au cas stationnaire :

Lecture des données : géométrie, matrice de compliance, viscosité, vitesse, ...

Initialisation du problème : calcul de $h(x, y)$ et du premier champ de pression.

Tant que les zones actives et inactives, épaisseur $h$, pression $p$ non stables.

Tant que partition $(\Omega)$ est non stable.

Problème de type 1 :

Calcul de $D$ (équation de Reynolds modifiée).

Mise à jour de la partition :

Si $D<0$ le nœud est éventuellement passé à l'état inactif;

Si $D \geq 0$ le nœud est éventuellement passé à l'état actif.

Fin.

Tant que les résidus $(h, p)>\varepsilon$ (méthode de NewtonRaphson).

Calcul des résidus des équations (Reynolds et charge appliquée).

Si premier passage ou si taux de convergence $<0,9$

calcul et triangularisation du Jacobien.

Résolution du système obtenu.

Correction de la pression $p$ et des paramètres de déplacement.

Calcul de la déformée élastique $h e$.

Modification de l'épaisseur du film $h$.

Fin

Fin

Écriture des résultats : pression, épaisseur du film, débit, puissance, ...

Fin de l'algorithme

\section{Résultats}

L'étude EHD que nous présentons concerne un joint à lèvre de diamètre $80 \mathrm{~mm}$ ayant un préserrage $2 \mathrm{~mm}$. La viscosité dynamique du fluide est de $\mu=0,031 \mathrm{~Pa} \cdot \mathrm{s}^{-1}$, le module de Young $E=7,5 \mathrm{MPa}$. et le coefficient de Poisson $\nu=0,49$. La fréquence de rotation varie de 500 à $6000 \mathrm{tr} \cdot \mathrm{min}^{-1}$.

Les caractéristiques calculées sont : les épaisseurs moyenne et minimale du film lubrifiant, la puissance dissipée, le champ d'épaisseur, le champ de pression et le débit.

On remarque sur la figure 5 que les épaisseurs moyenne et minimale du film lubrifiant varient peu avec la vitesse de rotation, comme l'avait déjà signalé Salant [12].

Sur la figure $6(\mathrm{a}, \mathrm{b}, \mathrm{c})$, on donne les répartitions globales de l'épaisseur du film lubrifiant (a), du champ de pression (b), et de la déformation de la lèvre (c), pour une fréquence de $6000 \mathrm{tr} \cdot \mathrm{min}^{-1}$. On constate que la répartition de l'épaisseur le long de la lèvre est globalement parabolique avec un minimum au centre de la cellule d'étude et un maximum sur les bords, et que

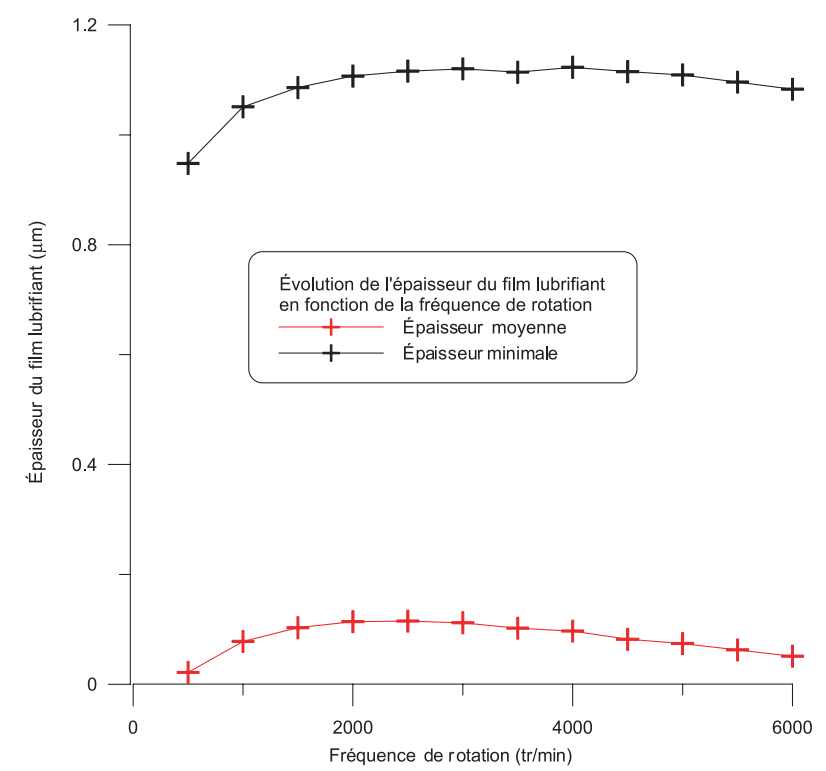

Fig. 5. Évolution des épaisseurs moyenne et minimale du film lubrifiant en fonction de la fréquence de rotation.

la déformation de la lèvre sous l'action de la pression présente un profil tridimensionnel conforme au champ de pression dont les zones actives ont une étendue restreinte. La résultante issue de ce champ équilibre parfaitement la charge due au préserrage.

La figure 7 montre que l'évolution de la puissance dissipée en fonction de la fréquence de révolution est parabolique, donc proportionnelle au carré de la fréquence de révolution. Les valeurs obtenues sont supérieures à celles observées réellement. En effet, cette dissipation d'énergie s'accompagne de l'accroissement de la température du lubrifiant sous la lèvre et donc de l'abaissement de sa viscosité, ce qui entraînera la chute des performances du joint. Pour analyser ce problème, une étude en T.E.H.D. globale a été entreprise et a montré une amélioration sensible des résultats obtenus pour la puissance. Elle suppose que cette dernière est entièrement dissipée par frottement et se transforme en chaleur. Cette chaleur est évacuée dans l'arbre par conduction et dans le fluide par convection.

Sur la figure 8, on compare les puissances dissipées en isotherme, avec celles obtenues avec l'hypothèse TEHD global. On constate que l'analyse TEHD globale permet de diminuer fortement la puissance dissipée.

Cela est dû à la décroissance exponentielle de la viscosité avec la température, laquelle atteint presque $136^{\circ} \mathrm{C}$, ce qui conduit à une viscosité de $3,85 \times 10^{-3} \mathrm{~Pa}$.s pour une fréquence de rotation de $6000 \mathrm{tr} \cdot \mathrm{min}^{-1}$. Cette valeur de la température n'est pas très éloignée de celle constatée expérimentalement (environ $120^{\circ} \mathrm{C}$ ).

\section{Conclusion}

Nous constatons une forte corrélation entre le champ de pression et les micro ondulations de la lèvre. La naissance et le maintien du film lubrifiant en dépendent. 


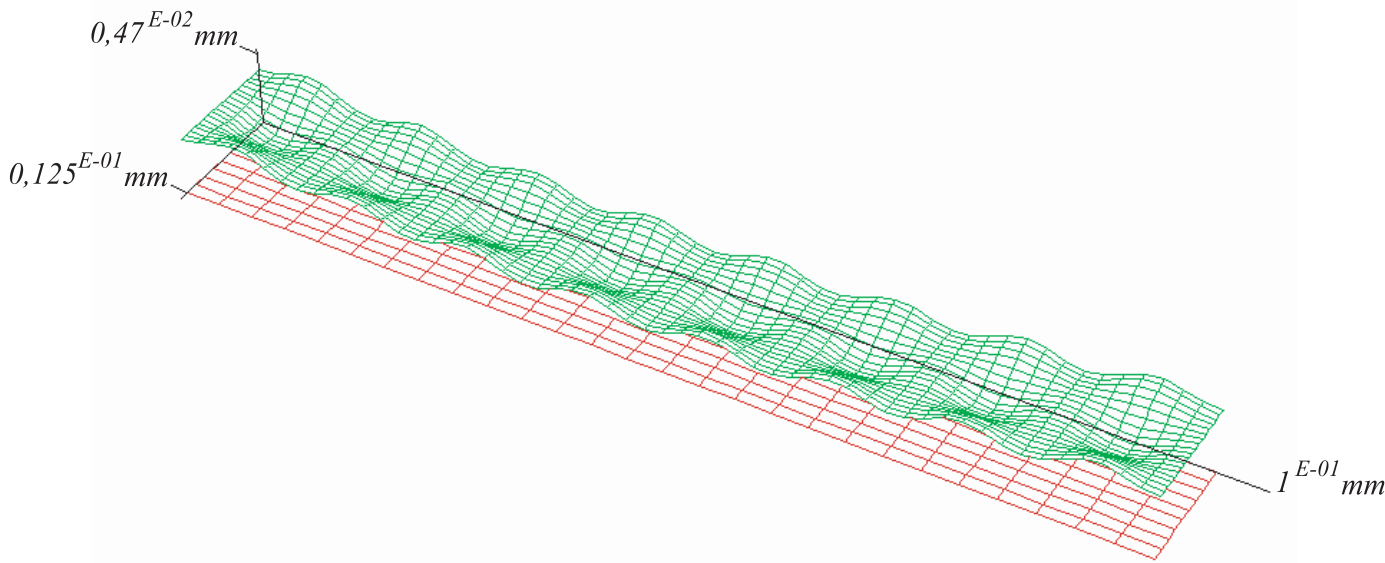

(a)

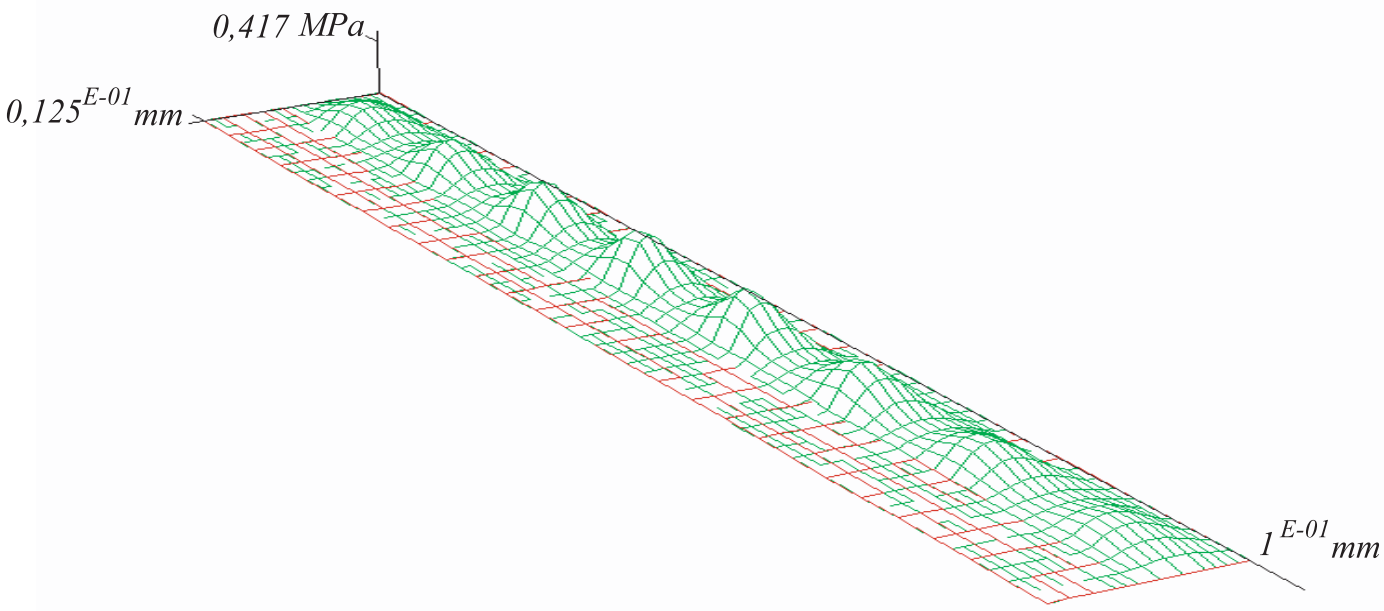

(b)

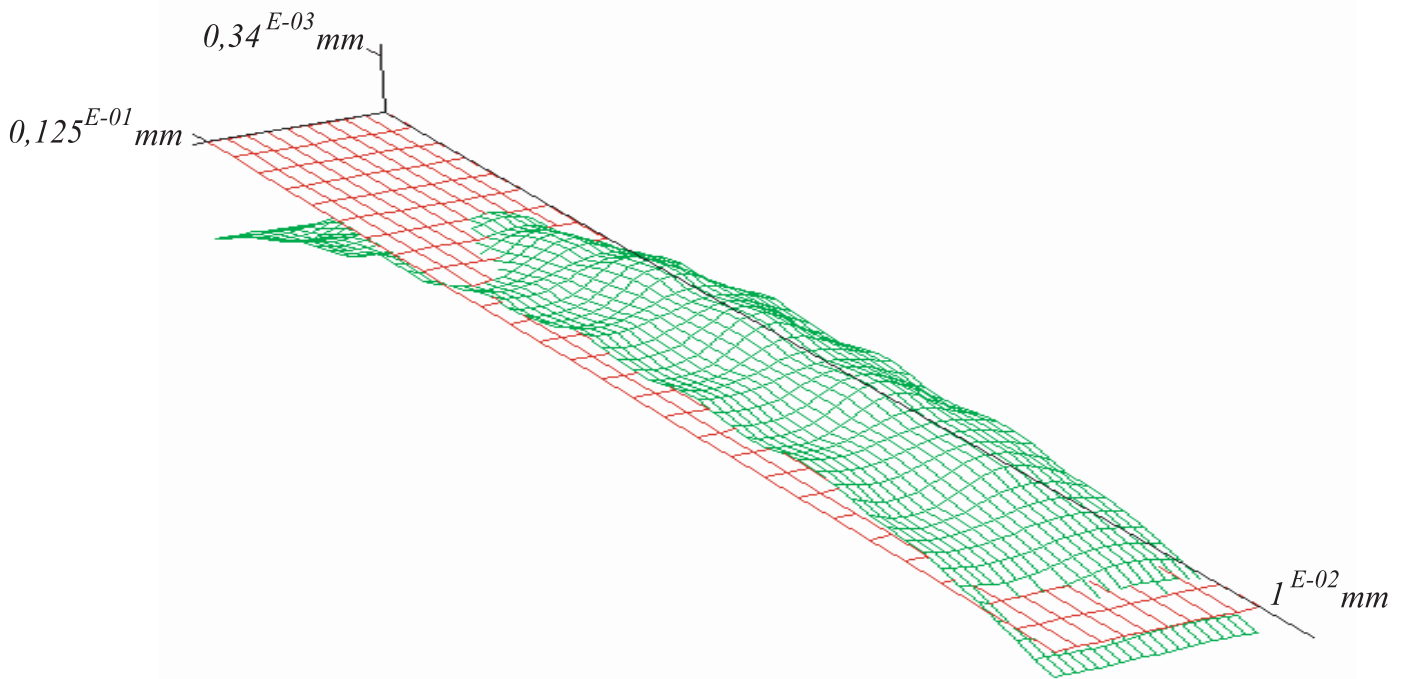

(c)

Fig. 6. (a) Épaisseur du film lubrifiant; (b) répartition de la pression; (c) déformation de la lèvre. 


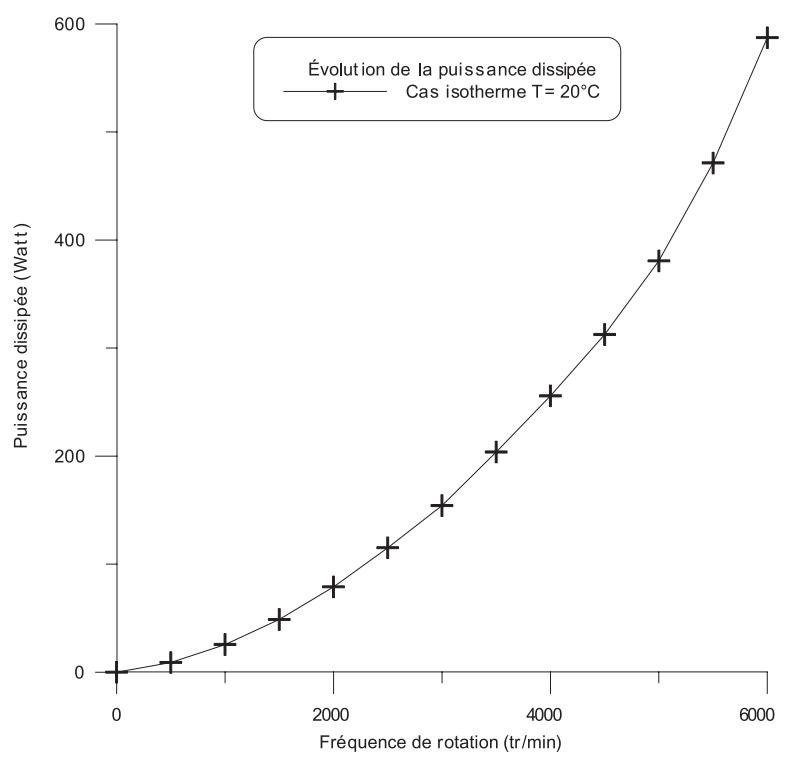

Fig. 7. Évolution de la puissance dissipée en fonction de la fréquence de rotation.

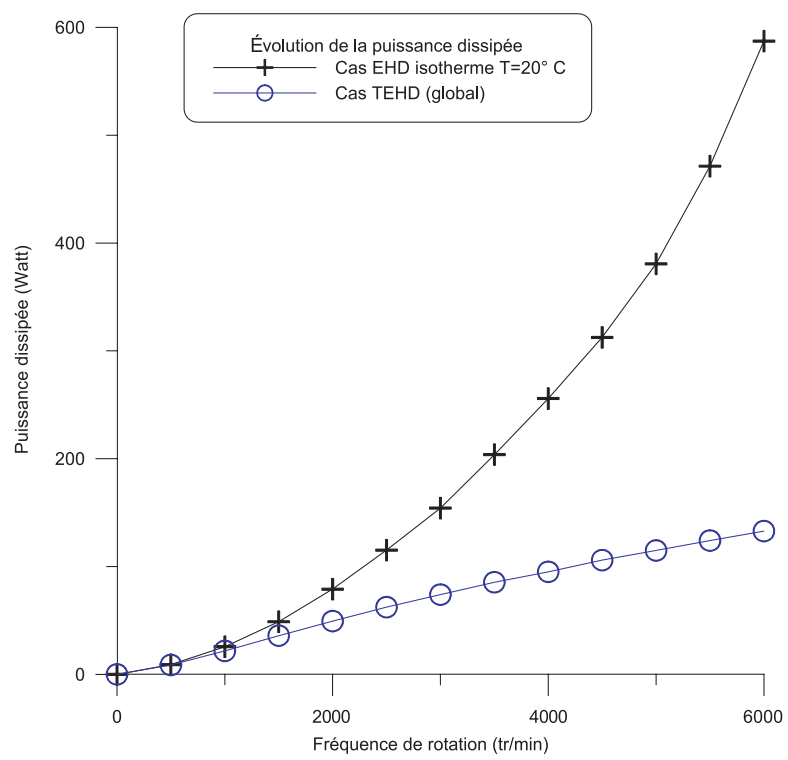

Fig. 8. Comparaison des puissances dissipées; cas isotherme et hypothèse TEHD global en fonction de la fréquence.

Ce champ de pression permet d'équilibrer la charge supportée par le joint. Il est directement lié aux grandeurs telles que la viscosité du fluide, le diamètre et la vitesse de l'arbre tournant, la température d'utilisation, le module de Young et le coefficient de Poisson du joint et l'état de surface de la lèvre.

Pour calculer les caractéristiques réelles de cet organe, il faut intégrer toutes ces données dans un processus qui ne peut être que ThermoElastoHydroDynamique (T.E.H.D.). Ce genre de formulation est complexe et difficile à mettre au point. Le logiciel réalisé intègre déjà la plupart de ces grandeurs. Il sera par la suite étendu au cas du TEHD local, ce qui nous permettra de modéliser avec précision cet organe.
Remerciements. Les auteurs remercient la Société Hutchinson pour son support technique et sa contribution financière à cette étude.

\section{Références}

[1] E.T. Jagger, Study of the lubrication of synthetic rubber rotary shaft seals, Proceedings, Conference on Lubrication and Wear, Inst. of Mech. Eng. (1957) 409-415

[2] E.T. Jagger, Further studies of lubrication of synthetic rubber rotary shaft seals, Proceedings, Inst. of Mech. Eng. 181 (1966) 191-204

[3] H.K. Müller, Concepts of sealing mechanism of rubber lip type rotary shaft seals, Proceedings, in : N.S. Nau (Ed.), 11th International Conference on Fluid Sealing, 1987, pp. 698-709

[4] L. Horve, A microscopic view of the sealing phenomenon for radial lip oil seals, Proceedings, N.S. Nau (Ed.), 11th International Conference on Fluid Sealing, 1987, pp. $710-731$

[5] L. Horve, Understanding the sealing mechanism of the radial lip seal for rotating shafts, Proceedings, N.S. Nau, (Ed.), 13th International Conference on Fluid Sealing, 1992, pp. 5-20

[6] H.K. Müller, G.W. Ott, Dynamic sealing mechanism of rubber rotary shaft seals, Proceedings, in : N.S. Nau (Ed.), 10th International Conference on Fluid Sealing, 1984, pp. 451-466

[7] K. Nakamura, Y. Kawahara, An investigation of sealing properties of lip seals through observations of sealing surfaces under dynamic condition, Proceedings in : N.S. Nau (Ed.), 10th International Conference on Fluid Sealing, 1984, pp. 87-105

[8] K. Nakamura, Sealing mechanism of rotary shaft lip-type seals, Tribology International 20 (1987) 90-101

[9] A. Gabelli, G. Poll, Formation on lubricant film in rotary sealing contacts part I lubricant film modeling J. Trib. 114 (1992) 280-289

[10] F. Shi, R. Salant, Numerical study of rotary lip seal with quasirandom sealing surface, J. Trib. 29 (2000) 1-8

[11] M.J.L. Stakenborg, On the sealing mechanism of radial lip seals, Tribology International 21 (1988) 335-340

[12] R. Salant, Elastohydodynamic model of the rotary lip seal, Trans. of the ASME, 118 (1996) 292-296

[13] R. Salant, A.L. Flaherty, Elastohydrodynamic analysis of reverse pumping in rotary lip seals with microasperities, J. Tribol. 116 (1995) 53-59

[14] K. Day, R.F. Salant, Thermal elastohydrodynamic model of a radial lip seal Part I : analysis and base results, J. Tribol. 121 (1999) 1-10

[15] B. Jacobson, L. Floberg, The finite Journal Bearing Considering Vaporisation, Chalmers Tekniska Hoegskolas Hundlingar 190 (1957) 1-116

[16] K. Olsson, On Hydrodynamic lubrication with special reference to nonstationary cavitation, Chalmers University of technology Goteborg, 1974

[17] D. Bonneau, M. Hajjam, Modélisation de la rupture et de la reformation des films lubrifiants dans les contacts élastohydrodynamiques, Revue européenne des éléments finis 10 (2001) 679-704 